\title{
Consistency of microvascular and autonomic abnormalities in diabetes
}

\author{
F H Karachaliou, K Karavanaki, R Greenwood, H Morgan, J D Baum
}

\begin{abstract}
The progression of early measures of microvascular disease and autonomic neuropathy were studied in a group of 81 children with insulin dependent diabetes mellitus over a mean interval of 4.2 years. Repeated measurements were made of blood pressure, albumin excretion, joint mobility, and pupillary dilatation in darkness. Over the years between the first and the second study, systolic and diastolic blood pressure showed positive tracking correlations $(r=0.38$ and $r=0.32)$ with a small but significant deviation from normality; albumin/creatinine ratio was significantly increased $(0.79 v 0.55)$; a greater number of children were identified in the second study as having limitation of mobility of the fifth metacarpophalangeal joint; and pupillary dilatation in darkness significantly decreased $(61.5 \%$ v $62.9 \%)$; $62 \%$ of the children with one or more abnormal measurements in the first study were found to have measurements outside the normal ranges in the second study, indicating a consistency in observations over time. It remains to be seen with what accuracy these measurements predict adult onset clinical disease.

(Arch Dis Child 1996;75:124-128)
\end{abstract}

Keywords: insulin dependent diabetes, blood pressure, microvascular abnormalities, joint mobility.

Diabetic complications first become clinically apparent during the second and third decades of life. However, microvascular ${ }^{1-4}$ and autonomic ${ }^{5}$ abnormalities may be demonstrable in children with relatively short diabetes duration. It is uncertain whether such findings remain consistent in individuals and with what accuracy they predict adult onset clinical disease. Such information is essential if progress is to be made in targeted intervention trials in childhood on the prevention of the complications of diabetes.

We report here a study of a cohort of children with diabetes, a proportion of whom show abnormalities which are consistent over time.

Department of Neurophysiology, Royal Hospital for Sick Children, Bristol H Morgan

Correspondence to: Dr F H Karachaliou, 5 Ulof Palme Ave, Ilissia 15771, Athens, Greece.

Accepted 24 April 1996
In 1986, a longitudinal study was initiated to document early markers of microvascular disease and autonomic neuropathy in diabetic children living in the population geographically defined by Avon County, United Kingdom.
Measurements of blood pressure, urinary albumin excretion, and joint mobility were performed on two occasions seven months apart, and groups of children with abnormal measurements on both these occasions were identified (study I). Pupillary dilatation in darkness, as a marker of autonomic dysfunction, was added to study I as part of the second set of measurements; $81(63 \%)$ of the same group of children were reassessed four years later (study II) to evaluate the consistency of findings and identify any children showing persistent abnormalities.

\section{SUBJECTS}

Eighty one of the original cohort of 129 diabetic children were reassessed after approximately four years (mean interval 4.2 years, range 3.4-4.8 years). At the time of study $I$ their age ranged from 3.7 to 16.8 years (mean 11.2 years) and duration of diabetes from 0.1 to 12.6 years (mean 3.8 years). Of the 48 who were not reassessed in study II, nine had moved away from Avon and 39 declined to take part. These 48 were older than those who agreed to participate (13.0 $v 11.2$ years) but did not differ significantly in other characteristics.

Control measurements were obtained from 121 (in study I) and from 65 (in study II) age and sex matched healthy children (the diabetic subjects' best friends).

\section{BLOOD PRESSURE}

Blood pressure was measured in the right arm with the child seated, using a random zero sphygmomanometer with the appropriate sized cuff, taking the fifth Korotkoff sound as the diastolic blood pressure. As blood pressure normal ranges are age and sex dependent, results were expressed as a SD score related to the 1987 Task Force on Blood Pressure Control data ${ }^{7}$ :

Blood pressure SD score $=$ (blood pressure measurement - mean)/SD,

where mean and SD are derived from the normal age and sex matched population.

\section{URINE ALBUMIN EXCRETION}

Urinary albumin excretion was measured on overnight urine samples. These were selected in preference to 24 hour or daytime collections because the intraindividual variability has been shown to be minimal. ${ }^{8}$ Urine albumin concentration was measured by the immunoturbidimetric assay (using the Cobas test) with an intra-assay coefficient of variation (CV) of $4 \%$, an interassay $\mathrm{CV}$ of $6 \%$, and a sensitivity of 0.5 
Table 1 Data on control children in studies I and II

\begin{tabular}{lll}
\hline Variables & Study $I(n=121)$ & Study $I(n=65)$ \\
\hline Age (years) (mean, SD) & $11.9(3.4)$ & $15.6(3.6)$ \\
Sex & $52: 69$ & $29: 38$ \\
Systolic BP SD score (mean, SD) & $0.23(0.8)$ & $0.13(0.7)$ \\
Diastolic BP SD score (mean, SD) & $0.23(0.8)$ & $0.14(0.7)$ \\
Pupil diameter \% (mean, SD) & $65.2(4.3)$ & $64.8(4.0)$ \\
ACR (mg/mmol) (geometric mean, 95\% & $0.49(0.15$ to 1.60$)$ & $0.47(0.14$ to 1.57$)$ \\
tolerance limits) & &
\end{tabular}

$\mathrm{BP}=$ blood pressure; $\mathrm{ACR}=$ albumin/creatinine ratio.

$\mathrm{mg} / \mathrm{l}$. Urine creatinine was measured by the Jaffe reaction. Urine albumin excretion was expressed as the geometric mean urine albumin/creatinine ratio in $\mathrm{mg} / \mathrm{mmol}$ of two consecutive overnight urine samples.

\section{JOINT MOBILITY}

The prayer sign $^{10}$ was used to examine the presence of limited joint mobility. In addition, each child was asked to place both hands down with fingers fanned on a desk top and the ability to appose the fingers to the flat surface was assessed. Children were classified in stages 0-III as follows:

Stage 0: if they were able to make complete approximation;

Stage I: if they were unable to make contact with some portion of a finger of one hand, usually the metacarpophalangeal (MCP) or the proximal interphalangeal (PIP) joint of the fifth finger;

Stage II: if they could not make contact with PIP or MCP joint of the fifth finger of both hands;

Stage III: if two IP joints were affected and/or more than one finger was affected.

Only children in stage II or III were considered as abnormal.

\section{PUPILLARY DILATATION}

Each child had the right eye photographed using a Polaroid pupillometer. The apparatus and photographic technique were identical to that described by Smith et al. ${ }^{9}$ The pupil and iris diameters were measured from the photograph and the pupil expressed as a percentage of the iris diameter using the formula:

Pupil diameter \% (PD\%) = (pupil diameter/ iris diameter) $\times 100$.

\section{GLYCAEMIC CONTROL}

This was assessed by glycated haemoglobin $\left(\mathrm{HbA}_{\mathrm{lc}}\right.$ measurements at every routine clinic visit (every three to four months) over the period of two years preceding each dataset collection.

$\mathrm{HbA}_{1 \mathrm{c}}$ was measured by an electrophoretic method, the normal range being 5.5-7.5\% The same laboratory method was employed for study I and study II

All the clinical measurements made in study II were made by one observer (FK) 'blind' to the results from study $I$ (made by $\mathrm{KK}$ ) and only after analysis were the data from the two studies linked together.

\section{STATISTICAL METHODS}

Computation and statistical analysis was performed using the SPSS system. Albumin/
Table 2 Characteristics the 81 diabetic children followed up (mean, SD)

\begin{tabular}{lcc}
\hline Variables & \multicolumn{1}{l}{ Study $I$} & \multicolumn{1}{l}{ Study II } \\
\hline Age (years) & $11.2(3.4)$ & $15.4(3.5)$ \\
Sex (M:F) & $33: 48$ & $33: 48$ \\
Duration (years) & $3.8(3.2)$ & $8.0(3.3)$ \\
HbA $_{1 \mathrm{c}}(\%)$ & $11.1(2.2)$ & $11.3(2.0)$ \\
Blood glucose (mmol/l) $_{\text {Insulin dose (U/kg/d) }}$ & $9.4(3.7)$ & $9.5(3.6)$ \\
& $0.80(0.2)$ & $0.94(0.3)$ \\
\hline
\end{tabular}

creatinine ratio values were logarithmically transformed to ensure normality. The distribution of all other values was found to be normal and conventional parametric statistics were used. Comparison of results between diabetics and controls or between abnormal and normal results of diabetic children were made by use of Student's $t$ test. Paired $t$ test was used for comparisons of values between the two studies. Associations between variables were examined using Pearson's correlation coefficients and $\chi^{2}$ analysis. A probability of less than $5 \%$ was taken as significant for all tests.

\section{Results}

Apart from age, there was no significant difference in the results for the control children between studies I and II (table 1).

The characteristics of the 81 diabetic children examined in both studies are shown in table 2 .

Mean (SD) $\mathrm{HbA}_{1 \mathrm{c}}$ concentration in study II was $11.3 \%$ (2.0) which was not different from that in study I (11.1\%) (mean difference: 0.24 ; SE: 0.25).

The tracking correlations for systolic and diastolic blood pressure between the first and second study were $r=0.38, \mathrm{p}<0.001$, and $r$ $=0.32, \mathrm{p}<0.01$, respectively (fig 1 ). Thus
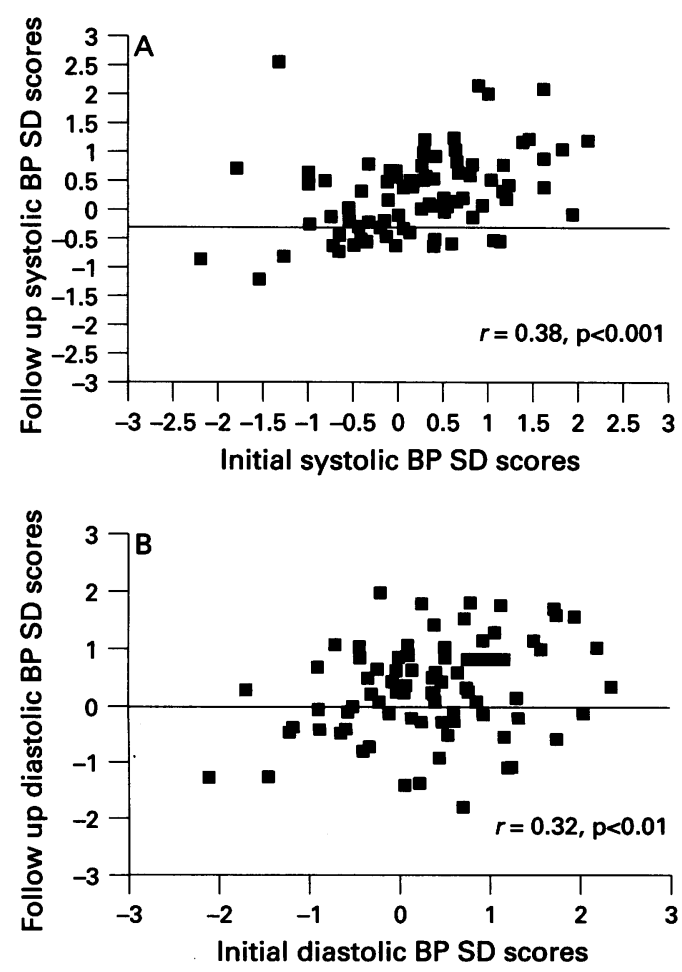

Figure 1 Relation of follow up to initial standardised scores $(A)$ for systolic and $(B)$ for diastolic blood pressure (BP). 
Table 3 Measurements of BP, ACR, PD\% and LFM of the 81 diabetic children followed up from study I and study II

\begin{tabular}{lllc}
\hline & Study I & Study II & p Value \\
\hline Systolic BP SD score (mean, SD) & 0.23 & 0.28 & 0.67 \\
Diastolic BP SD score (mean, SD) & 0.29 & 0.34 & 0.71 \\
ACR (mg/mmol) (geometric mean, 95\% tolerance limit) & $0.55(0.1$ to 2.88) & $0.79(0.09$ to 6.48$)$ & $<0.01$ \\
No of children with LJM (stage II/III) & $7 / 81$ & $14 / 81$ & $<0.05$ (McNemar's test) \\
Pupil diameter \% (mean, SD) & $62.9(4.5)$ & $61.5(4.5)$ & $<0.001$ \\
\hline
\end{tabular}

$\mathrm{BP}=$ blood pressure; $\mathrm{ACR}=$ albumin/creatinine ratio $\mathrm{LJM}=$ limited joint mobility.

there was a small but significant positive relation between the blood pressure measurements in study II and study I. The standard scores of both systolic and diastolic blood pressure in study II were, however, not significantly different from those in study I (systolic: $0.28 v$ 0.23 , mean difference 0.045 ; SE $0.1 ; \mathrm{p}=0.67$; diastolic: $0.34 v 0.29$, mean difference 0.046 ; SE $0.11 ; \mathrm{p}=0.71$ ).

Other study II measurements were compared with study I (table 3): the geometric mean (95\% confidence interval) for the albumin/creatinine ration was $0.79(0.09$ to 6.48$) \mathrm{mg} / \mathrm{mmol}$, which was significantly greater than that in study $I, 0.55$ (0.1 to 2.88 ) $\mathrm{mg} / \mathrm{mmol}$ (mean difference 0.72 ; SE 1.14; $p<0.01$ ).

The number of children with limited joint mobility in study II was double that in study I $(14 / 81 v 7 / 81$, McNemar's test $=5.4, \mathrm{p}<0.05)$.

Pupillary adaptation in darkness in study II was $61.5 \%$ (4.5), which was significantly reduced compared with that of study I-62.9\% (4.5) (mean difference 1.43; SE 0.24; $\mathrm{p}<0.001$ ).

There was a close correlation between the measurements of pupillary dilatation in the two studies $(r=0.94, \mathrm{p}<0.001$ ) (fig 2). The decrease in PD\% could not be attributed to age, as PD\% was not associated with age in either diabetic or control children in study I or study II. In diabetic children there was a significant negative correlation between PD\% and measurements of glycaemic control $(r=$ $-0.40, \mathrm{p}<0.01)$ and a weaker association between PD\% and duration of disease $(r=$ $-0.25, \mathrm{p}<0.05)$.

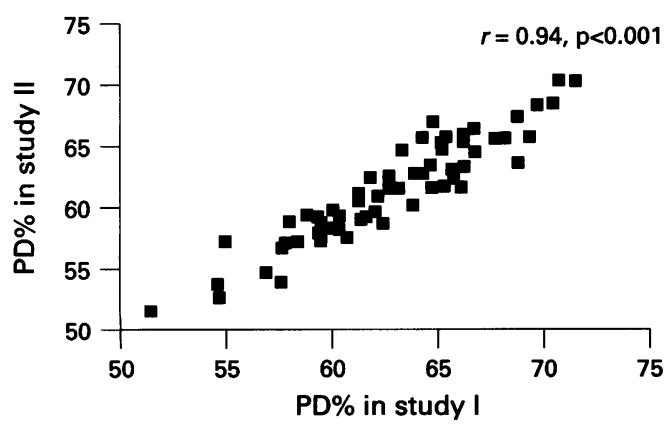

Figure 2 Scattergram comparing PD\% results between the two studies.

Table 4 Consistency of abnormal measurements

\begin{tabular}{lllcl}
\hline & \multicolumn{4}{l}{ Children with abnormalities in } \\
\cline { 2 - 5 } & Neither of the studies & Study I only & Study II only & Both studies \\
\hline Blood pressure & 62 & 12 & 7 & 5 \\
Albumin/creatinine & 59 & 12 & 10 & 9 \\
Limited joint mobility & 66 & 7 & 8 & 6 \\
Pupillary dilatation \% & 72 & 5 & 4 & 5 \\
\hline
\end{tabular}

CONSISTENCY OF ABNORMAL MEASUREMENTS In study I a group of 27 children was identified as having one or more abnormal measurements. In study II, 17 of these 27 children (63\%) again showed abnormal values for the same or additional tests (table 4).

\section{Blood pressure}

Of the 12 children who had raised blood pressure in two sessions of measurements in study I, five $(42 \%)$ were found to be above the defined confidence intervals for age and sex in study II. An additional seven children were identified with abnormal blood pressure measurements in study II.

\section{Urine albumin excretion}

Of the 12 children with albumin/creatinine ratio values above the normal range on both sessions in study $I$, nine $(75 \%)$ were found again above the normal range. An additional 10 children were found to have abnormal values in study II.

\section{foint mobility}

Of seven children identified as having stage II or III of joint limitation in study I, six (86\%) and an additional eight children were shown to have joint limitation in study II.

\section{Pupillary dilatation}

All five children identified as having abnormal PD\% in study I had PD\% below the normal range defined from the control group in study II. An additional four children were below the normal values in study II.

\section{COEXISTENCE OF ABNORMALITIES}

Previous studies have shown a high rate of concordance between specific diabetic complications. ${ }^{11}$ The nine children identified in both studies with a raised albumin/creatinine ratio were all more than 15 years old, all had a urine albumin excretion of $>15 \mu \mathrm{g} / \mathrm{min}$, but none had frank albuminuria (urine albumin excretion $>200 \mu \mathrm{g} / \mathrm{min}$ ). One child from study I also had a raised blood pressure. In study II he was also found to have both a raised albumin/ creatinine ratio and raised blood pressure. An additional two of the children with a raised albumin/creatinine ratio were found to have raised blood pressure in study II.

In study II both systolic and diastolic blood pressure scores of the group of children with a raised albumin/creatinine ratio were significantly higher than those of the rest of diabetic children, whereas their blood pressure scores in the first study were not different from the rest of diabetic children. 
The degree of coexistence of abnormalities noted in study I was more evident in study II, with the number of children with more than one abnormal measurement being significantly greater $(15 / 78 v 6 / 78$, McNemar's test $=9$, $\mathrm{p}<0.01)$.

Thus in study I two children had three abnormalities: one of these had abnormal measurements on all four tests in study II. There were four children in study I with two abnormalities; in study II three of them had an additional abnormal measurement. Another nine children had more than one abnormality in study II.

\section{Discussion}

Subclinical changes in renal function, ${ }^{1-3}$ blood pressure, ${ }^{4}$ and autonomic function ${ }^{56}$ in diabetic children have been reported even in early childhood. However, it is uncertain whether these changes are consistent over time and can serve as markers for the development of long term complications of diabetes.

In healthy children and adolescents, the capacity of a given blood pressure measurement to predict subsequent blood pressure has been studied using correlation coefficients between repeated measurements at different time intervals and has been shown to be rather limited. ${ }^{12}{ }^{13} \mathrm{~A}$ similar low degree of blood pressure tracking has been reported in children with diabetes, ${ }^{14}$ suggesting that it is difficult to predict future blood pressure levels for individual patients. In agreement with these studies, we found only a small tracking correlation coefficient of blood pressure measurements repeated after four years in a cohort of 81 children with diabetes. Furthermore, of the 12 children with raised blood pressure in both sessions of measurements in study I, only five were shown to be above the defined confidence intervals for age and sex some three years later.

Microalbuminuria, that is, urinary albumin excretion which while raised is yet below the level of Albustix-positive albuminuria, has been shown in adults with diabetes to predict nephropathy. ${ }^{15} 16$ Microalbuminuria has been shown to be present in $12-20 \%$ of diabetic children. ${ }^{23}$ However, there is a high day to day variation in urinary albumin excretion ${ }^{8}$ and repeated measurements need to be performed before classifying patients as having abnormal or normal values. In study I, four 24 hour urine collections were performed, two at the start of the study and another two 6-7 months later. Only the 12 children with abnormal values in both periods were then classified as having persistent microalbuminuria. In study II, while none of these children had developed clinical proteinuria, nine $(75 \%)$ still had values outside the normal range. Overall, the correlation between measurements in the two studies was small $(r=0.28)$.

The upper normal $95 \%$ tolerance limit for albumin/creatinine ratio in our study was estimated at $1.57 \mathrm{mg} / \mathrm{mmol}$. There are very limited published data on normal values for the albumin/creatinine ratio or urinary albumin excretion in children. Davies $e t a l^{17}$ defined the upper normal range for the albumin/creatinine ratio at $1.72 \mathrm{mg} / \mathrm{mmol}$ for girls and 1.57 $\mathrm{mg} / \mathrm{mmol}$ for boys, in 12 hour night urine collections from 374 normal children. Gibb et $a l^{18}$ also reported the upper normal limit of the range for the albumin/creatinine ratio to be $1.17 \mathrm{mg} / \mathrm{mmol}$ in 45 healthy children.

Limited joint mobility, a relatively common phenomenon in childhood diabetes, was detected more commonly in study II than in study I, and a high degree of coexistence with other abnormalities was noted. ${ }^{19}$

Reduced pupil size in darkness, believed to be due to autonomic neuropathy, ${ }^{20}$ has been shown to be common in patients with insulin dependent diabetes mellitus. ${ }^{21}$ Moreover pupil size has been used in addition to cardiovascular tests as a measure of autonomic function. ${ }^{22} \mathrm{We}$ found a close correlation $(r=0.94)$ between measurements of pupillary dilatation in darkness performed in the two studies using the same technique. Among the five children with abnormal values in study I none had developed any clinical symptoms of autonomic neuropathy, but all five had abnormal pupillary response to darkness in study II. For the group as a whole, PD\% in study II was found to be significantly decreased compared with study I. This is likely to represent a diabetes effect, as the sympathetic dysfunction due to aging noted in non-diabetic populations is not demonstrable in childhood. ${ }^{6}$

In this cohort study we have identified a group of diabetic children and adolescents with persistent abnormalities. However, 15 children and adolescents were identified (M/F $6 / 9$; mean age 15.7 years; disease duration 7.8 years), who despite having a mean $\mathrm{HbA}_{1 \mathrm{c}}$ of $>$ $10 \%$ throughout the period between the two studies, did not have any measurable abnormalities in either study.

We conclude that early subclinical changes of renal and autonomic function in a cohort of children with diabetes reassessed after four years were (with the exception of blood pressure measurements) relatively consistent, with some pointers to a progression in the number of children showing abnormalities and the degree of abnormality. Further follow up is necessary to establish wether these findings reliably predict the long term development of clinical complications. If this proves to be the case then the tests could be used to identify patients for trials of targeted treatment to improve glycaemic control or alternative interventions such as the use of angiotensin converting enzyme inhibitors. Conversely, it remains to be shown whether persistently normal test results identify a population of patients who, despite indifferent control, appear to be at a relatively lower risk of developing the complications of diabetes: were this to be the case it might justify a different and more relaxed approach to glycaemic control in such children.

This work was supported by the Choc Wilson Scholarship, Faculty of Medicine, University of Bristol.

\footnotetext{
1 Ellis D, Becker DJ, Daneman D, Lobes L, Drash A. Proteinuria in children with insulin-dependent diabetes: relationship to duration of disease, metabolic control, and retinal changes. $\mathcal{f}$ Pediatr 1983;102:673-80.
} 
2 Mathiesen ER, Saurbrey N, Hommel E, Parving HH. Prevalence of microalbuminuria in children with type 1 (insulin-dependent) diabetes mellitus. Diabetologia 1986; 29:640-3.

3 Dalquist G, Rudberg S. The prevalence of microalbuminuria in diabetic children and adolescents and its relation to ria in diabetic children and adolescents and its

puberty. Acta Paediatr Scand 1987;76:795-800.
4 Tarn AC, Drury PL. Blood pressure in children, adolescents and young adults with type 1 (insulin-dependent) diabetes. Diabetologia 1986;29:275-81

5 Mithcell EA, Wealthall SR, Elliot RB. Tests of autonomic neuropathy in diabetic children. Aust Paediatr $\mathcal{F}$ 1985; 21:105-9.

6 Clarke CF, Piesowicz AT, Spathis GS. Pupillary size in children and adolescents with type 1 diabetes. Diabet Med 1989;6:780-3.

7 Task Force on Blood Pressure Control in Children. Report of the Second Task Force on Blood Pressure Control in Children-1987. Pediatrics 1987;79:1-25.

8 Hutchison AS, Paterson KR. Collecting urine for microalbumin assay. Diabet Med 1988;5:527-32.

9 Smith SA, Dewrist RR. A simple diagnostic test for pupillary abnormality in diabetic autonomic neuropathy. pupillary abnormality in diabe
Diabet Med 1986;66:1085-92.

10 Rosenbloom AL, Silverstein JH, Lezotte DC, Richardson K, McCallum M. Limited joint mobility in childhood diabetes mellitus indicates increased risk for microvascular disease. N Engl f Med 1981;305:191-4.

11 Orchard TJ, Dorman JS, Maser RE, et al. Prevalence of complications in IDDM by sex and duration. Pittsburgh Epidemiology of Diabetes Complications Study II. Diabetes 1990;39:1116-24.

12 Rosner B, Hennekens CH, Kaas EH, Miall WE. Age specific correlation analysis of longitudinal blood pressure data. Am $\mathcal{F}$ Epidemiol 1977;106:306-13.
13 Berenson GS, Voors AW, Webber LS, Frerichs RR. Blood pressure in children and its interpretation. Pediatrics 1978; 61:333-6.

14 Ibsen KK, Rotne H, Hougaard P. Tracking of blood pressure in diabetic children. Acta Paediatr Scand 1983; 72:495-8.

15 Viberti GC, Hill RD, Jarrett RJ, Argyropoulos A, Mahmud $\mathrm{U}$, Keen H. Microalbuminuria as a predictor of clinical nephropathy iin insulin-dependent diabetes mellitus. Lancet 1982;i:1430-2.

16 Morgensen CE, Christiansen CK. Predicting diabetic nephropathy in insulin-dependent patients. $N$ Engl f Med 1984; 311:89-93.

17 Davies AG, Postlethwaite RJ, Price DA, Burn JL, Houlton CA, Fieldig BA. Urinary albumin excretion in school chilCA, Fieldig BA. Urinary albumin excreti
dren. Arch Dis Child 1984;59:625-30.

18 Gibb DM, Tomlinson P, Dalton NR, Turner C, Shah V, Barratt TM. Renal tubular proteinuria and microalbuminuria in diabetic patients. Arch Dis Child 1989;64:12934.

19 Brink SJ. Limited joint mobility as a risk factor for comlications in youngsters with insulin-dependent diabetes mellitus. In: Pediatric and adolescent diabetes mellitus. Chicago: Year Book Medical Publishers, 1987:305-12.

20 Smith SA, Smith SE. Evidence for a neuropathic aetiology in the small pupil of diabetes mellitus. Br $₹$ Ophthalmol in the small pupil.

21 Hreidarsson AB. The pupil of the eye in diabetes mellitus, an indicator of autonomic nervous dysfunction. Danish Med Bull 1992;39:400-8.

22 Ziegler D, Cicmir I, Mayer P, Wiefels K, Gries FA. The natural course of peripheral and autonomic neural function during the first two years after diagnosis of type 1 diabetes. Klin Wochenschr 1988;66:1085-92. 